\title{
Simultaneous polarization monitoring of supernovae SN 2008D/XT 080109 and SN 2007uy: isolating geometry from dust
}

\author{
J. Gorosabel ${ }^{1}$, A. de Ugarte Postigo ${ }^{2,3}$, A. J. Castro-Tirado ${ }^{1}$, I. Agudo ${ }^{1,4}$, M. Jelínek ${ }^{1}$, S. Leon ${ }^{5}$, T. Augusteijn 6 , \\ J. P. U. Fynbo ${ }^{7}$, J. Hjorth ${ }^{7}$, M. J. Michałowski ${ }^{7}$, D. Xu ${ }^{7}$, P. Ferrero ${ }^{8,9,10}$, D. A. Kann ${ }^{10}$, S. Klose ${ }^{10}$, A. Rossi ${ }^{10}$, \\ J. P. Madrid ${ }^{11}$, A. LLorente ${ }^{12}$, M. Bremer ${ }^{13}$, and J.-M. Winters ${ }^{13}$ \\ 1 Instituto de Astrofísica de Andalucía (CSIC), Glorieta de la Astronomía s/n, 18008 Granada, Spain \\ e-mail: jgu@iaa.es \\ 2 European Southern Observatory, Casilla 19001, Santiago 19, Chile \\ 3 INAF/Osservatorio Astronomico di Brera, via Bianchi 46, 23807 Merate, LC, Italy \\ ${ }^{4}$ Institute for Astrophysical Research, Boston University, 725 Commonwealth Avenue, Boston, MA 02215, USA \\ 5 Joint ALMA Observatory, Av. El Golf 40, Piso 18, Las Condes, Santiago de Chile, Chile \\ ${ }^{6}$ Nordic Optical Telescope, Apartado 474, 38700 Santa Cruz de La Palma, Spain \\ 7 Dark Cosmology Centre, Niels Bohr Institute, University of Copenhagen, Juliane Maries Vej 30, 2100 Copenhagen $\emptyset$, Denmark \\ 8 Instituto de Astrofísica de Canarias, vía Láctea s/n, 38200 La Laguna, Tenerife, Spain \\ 9 Departamento de Astrofísica, Universidad de La Laguna (ULL), 38205 La Laguna, Tenerife, Spain \\ 10 Thüringer Landessternwarte Tautenburg, Sternwarte 5, 07778 Tautenburg, Germany \\ 11 Centre for Astrophysics \& Supercomputing, Swinburne University of Technology, PO Box 218, Hawthorn, VIC 3122, Australia \\ 12 INSA, Herschel Science Operations Centre, European Space Agency, Villafranca del Castillo, PO Box 50727, 28080 Madrid, \\ Spain \\ 13 Institute de Radioastronomie Millimétrique (IRAM), 300 rue de la Piscine, 38406 Saint Martin d'Hères, France
}

Received 7 September 2009 / Accepted 25 June 2010

\section{ABSTRACT}

Context. The possible existence of a continuum encompassing the diversity of explosive stellar deaths, ranging from ordinary supernovae (SNe; lacking any sign of a relativistic outflow) to relativistic hypernovae associated with energetic long duration gamma-ray bursts (GRBs), is under intense debate. In this context, the supernova SN 2008D associated with the X-ray transient (XT) 080109 could represent a paradigmatic case, since it might exemplify a potential borderline transition event. Optical polarimetric studies could contribute to shed light on the different interpretations given in the literature for this supernova (hereafter, SN 2008D/XT 080109).

Aims. The main aim is to infer geometric information of SN 2008D/XT 080109 through the study of the evolution of its linear optical polarization. We also report the polarization evolution of SN 2007uy, and discuss the properties of the host galaxy interstellar medium (ISM) towards the XT. The final goal is to compare the polarization properties, and therefore the geometries, of both SNe.

Methods. We present a $V$-band linear polarization monitoring campaign carried out for SN 2008D/XT 080109 and SN 2007uy, which shone for weeks contemporaneously in NGC 2770. This fortunate coincidence brought us the opportunity to observe both objects simultaneously, and most importantly, with identical instrumental setups. The observations span 74.9 days, starting 3.6 days after the XT and are distributed in 11 visits. In addition we performed observations in the millimetre $(\mathrm{mm})$ range in order to identify the dominant origin of the observed polarization.

Results. We report positive linear polarization detections at several epochs for SN 2008D/XT 080109 at a level of $~ 1 \%$. For SN 2007uy the measured polarization is around $\sim 1.5 \%$. In both cases the observed linear polarization seems dominated by the host galaxy interstellar polarization (HGIP), especially for the case of SN 2007uy. SN 2007uy shows Stokes parametres consistent with no time evolution, which could be described by the HGIP plus a constant eccentricity expansion on the sky plane. Over the course of our observations of SN 2007uy, we find that its total polarization signal does not change by more than $0.29 \%$ with a $90 \%$ confidence interval. Despite the dominant HGIP, a statistical analysis of the distribution of the SN 2008D/XT 080109 Stokes parametres suggests that it could show a possible intrinsic variable polarization component. Moreover, assuming the polarization signal from SN 2007uy is constant, we find that the temporal evolution of the intrinsic SN 2008D/XT 080109 polarization could be explained by an aspherical axisymmetric expansion with variable eccentricity, although other more complex geometric scenarios are also compatible with the data. We come to the same result even if we make no assumption on the SN 2007uy Stokes parametres, although at a lower significance level.

Conclusions. We conclude that the data seem to suggest a potential symmetry axis for SN 2008D/XT 080109, which is reinforced when SN 2007uy is assumed to have constant Stokes parametres and used as reference star. We suggest that at least the projected, if not the intrinsic, geometry of SN 2008D/XT 080109 and SN 2007uy could be different.

Key words. supernovae: individual: SN 2008D/XT 080109 - supernovae: individual: SN 2007uy - techniques: polarimetric gamma-ray burst: general

\section{Introduction}

The X-ray transient (XT) 080109 was serendipitously discovered on 2008 January 9.56446 UT by Swift as a bright
X-ray outburst during a scheduled observation of SN 2007uy (Berger \& Soderberg 2008; Kong \& Maccarone 2008). Optical follow-up observations revealed the presence of a supernova (SN) associated with the XT (Deng \& Zhu 2008, hereafter 
named SN 2008D/XT080109). SN 2008D/XT080109 underwent a transition 1-2 weeks after the XT from type Ic to Ib (Malesani et al. 2009; Blondin et al. 2008; Valenti et al. 2008; Modjaz et al. 2009), resembling the spectral evolution seen in SN 2005bf (Anupama et al. 2005; Modjaz et al. 2005; Tominaga et al. 2005; Wang \& Baade 2005). Eventually, SN 2008D/XT 080109 was spectroscopically classified as a He-rich Ib supernova (Modjaz et al. 2009).

The origin of this XT is still unclear. Two main scenarios have been proposed to explain its nature; the stellar shock breakout (Soderberg et al. 2008; Chevalier \& Fransson 2008) and the X-ray flash framework (Li 2008; Xu et al. 2008; Mazzali et al. 2008). In the first case the early emission would be entirely explained by the supernova, whereas in the second case a relativistic outflow from a central engine would be required. Radio data seem to indicate no superluminal motions, arguing against the presence of a long-lived relativistic outflow (Bietenhloz et al. 2009), therefore supporting the first framework. However, different spectral analyses of the X-ray data did not yield conclusive results on the possible presence of a thermal component expected from the shock breakout wave (Chevalier \& Fransson 2008; Li 2008; Soderberg et al. 2008; Modjaz et al. 2009). Within the second scenario SN 2008D/XT 080109 was proposed as a transition between the most energetic hypernovae (linked to canonical long duration gamma-ray bursts) and standard corecollapse SNe (Mazzali et al. 2008). In fact it was suggested that SN 2008D/XT 080109 is a side-viewed, bipolar explosion with a viewing angle of $>50^{\circ}$ from the polar direction (Tanaka et al. 2009a). It should be noted that spectroscopic observations of SN 2008D/XT 080109 suggested an aspherical expansion geometry (Modjaz et al. 2009; Tanaka et al. 2009a).

SN 2007uy was discovered on 2007 Dec. 31.669 UT (Nakano et al. 2008). It was classified as an ordinary Ib SN (with no hint of any superluminal outflow) before the light curve maximum (Blondin \& Calkins 2008), showing spectral similarities with SN 2004gq (Pugh et al. 2004; Modjaz \& Falco 2005). In the optical both SN 2008D/XT 080109 and SN 2007uy shone contemporaneously for weeks in the nearby $(z=0.007)$ spiral galaxy NGC 2770. This fortunate coincidence brought us the opportunity to observe both objects simultaneously, and most importantly, with the same instrumental setup.

Recently Maund et al. (2009) have reported a spectropolarimetric study of SN 2008D/XT 080109 based on data acquired on 2008 January 31.22 and February 15.18 UT. One of the main advantages of spectropolarimetry of SNe with respect to broadband polarimetry is its ability to infer geometric and dynamical information for the different chemical constituents of the explosion. Broad-band polarimetric observations construct a rougher picture of the stellar death, but require lower signal-to-noise ratios than spectropolarimetry. Hence broad-band polarimetric observations can be extended to objects at higher redshifts or/and they allow to enhance the polarimetric coverage and sampling of the light curve, especially at epochs far from the maximum when the $\mathrm{SN}$ is dimmer.

The dominant intrinsic polarization in $\mathrm{SNe}$ is thought to originate by Thompson photon scattering through an aspherical photospheric expansion (Höflich 1991). If the SN photosphere projection on the sky plane is an ellipse, in general a non-cancelled linear polarization is expected perpendicular or parallel to the major axis (Kasen et al. 2003). Therefore, if the expansion ellipticity evolves with time, keeping the direction of both axes unchanged, then the Stokes parametres should also move on a straight line of the Stokes plane. Once the Milky Way Galactic interstellar polarization (GIP) has been corrected, this straight line is expected to be displaced from the origin of the Stokes plane due to the host galaxy interstellar polarization (HGIP). Using several different methods Maund et al. (2009) estimated a HGIP of $P \sim 1.2 \%$ and $\theta \sim 134^{\circ}$ for SN 2008D/XT 080109. The contribution of additional polarization components (variable line polarization, irruption of the stellar core, variable HGIP, etc.) can distort this rough ellipsoidal stellar picture, resulting in a more complex geometrical configuration (see Maund et al. 2009, and references therein). Asphericities in explosive stellar deaths tend to be low, usually yielding optical polarization values below $\sim 1 \%$ (Wang \& Wheeler 2008). However, counterexamples exist, such as the type Ic SN 1997X (Wang et al. 2001) or XRF 060218/SN2006aj (Gorosabel et al. 2006; Maund et al. 2007).

In this paper we report optical polarimetric observations of SN 2008D/XT 080109 and SN 2007uy aimed at obtaining geometrical information on the expansion of these events. In addition we show the results of millimetre $(\mathrm{mm})$ observations which were used to infer information about the host galaxy extinction and consequently about the HGIP impact on the observed polarization.

\section{Observations and data analysis}

\subsection{Optical observations}

Table 1 displays the log of our optical observations. All the data were taken in the $V$-band. The observations were carried out with the $2.2 \mathrm{~m}$ Calar Alto telescope (CAHA), the $2.5 \mathrm{~m}$ Nordic Optical Telescope (NOT) and the $8.2 \mathrm{~m}$ Unit Telescope 2 of the Very Large Telescope (VLT).

The NOT observations were performed with ALFOSC, through a calcite and a $1 / 2$ wave retarder plate. Four images of the field were acquired, rotating the retarder plate at $0^{\circ}, 22.5^{\circ}$, $45.0^{\circ}$ and $67.5^{\circ}$. The calcite plates reduce the ALFOSC field of view (FoV) to $140^{\prime \prime}$ in diameter (pixel scale of 0 . $^{\prime} 19 /$ pix). SN 2007uy was left out of the NOT images due to this reduced FoV.

The CAHA observations were based on the CAFOS instrument. The CAFOS polarization unit uses a Wollaston prism instead of a calcite plate and has a strip mask on the focal plane to avoid accidental overlapping on the CCD. The total FoV of CAFOS is composed by 14 strips of $9^{\prime} \times 18^{\prime \prime}$ each, providing a large enough FoV to image both SN 2008D/XT 080109 and SN 2007uy simultaneously.

The VLT observations were done with FORS1, with a setup similar to CAFOS. As in CAFOS, the large FoV of FORS1 allowed us to simultaneously image SN 2008D/XT080109 and SN 2007uy. In some of the FORS1 visits we used the $1 \times 1$ bin read-out mode in order to avoid saturation of SN 2007uy while keeping a high signal to noise ratio for SN 2008D/XT 080109. We note that, in addition to the CCD binning, the seeing and transparency conditions varied significantly from night to night. This made it impossible to have a bright unsaturated field star common to all our images. Apart from SN 2008D/XT 080109, SN 2007uy was the object most frequently imaged, being present in the FoV of all the FORS1 and CAFOS images. This fact provided us with the opportunity of using SN 2007uy as a polarimetric reference star on the Stokes plane (see discussion of Sect. 3.2).

The images were reduced using standard procedures running under IRAF ${ }^{1}$. The master flat field image was created

\footnotetext{
1 IRAF is distributed by the National Optical Astronomy Observatory, which is operated by the Association of Universities for Research in
} 
Table 1. Polarimetric observations of SN 2008D/XT 080109 and SN 2007uy.

\begin{tabular}{|c|c|c|c|c|c|c|c|c|c|}
\hline \multirow{2}{*}{$\begin{array}{c}\text { Date }^{a} \\
\text { 2008, UT }\end{array}$} & \multirow{2}{*}{$\begin{array}{c}\text { Telescope } \\
\text { (+Instrument) }\end{array}$} & \multicolumn{2}{|c|}{ Exposure Seeing } & \multicolumn{3}{|c|}{ SN 2008D/XT 080109} & \multicolumn{3}{|c|}{ SN 2007uy $^{b}$} \\
\hline & & time $(\mathrm{s})$ & $\left({ }^{\prime \prime}\right)^{\circ}$ & $P \pm \sigma_{P}(\%)$ & $\theta \pm \sigma_{\theta}(\mathrm{deg})$ & $V \pm \sigma_{V}^{c}$ & $P \pm \sigma_{P}(\%)$ & $\theta \pm \sigma_{\theta}(\operatorname{deg})$ & $V \pm \sigma_{V}{ }^{c}$ \\
\hline Jan. 13.1644 & NOT(+ALFOSC) & $12 \times 600$ & 0.7 & $0.95 \pm 0.20$ & $114.9 \pm 5.9$ & $18.44 \pm 0.05$ & - & - & - \\
\hline Jan. 13.2642 & VLT $(+$ FORS1) & $2330^{d}$ & 1.0 & $\begin{array}{c}0.68 \pm 0.22 \\
(0.65 \pm 0.33)^{e}\end{array}$ & $\begin{array}{c}119.2 \pm 9.5 \\
(124.5 \pm 13.3)\end{array}$ & $18.39 \pm 0.05$ & $\begin{array}{c}1.45 \pm 0.25 \\
(1.59 \pm 0.04)\end{array}$ & $\begin{array}{c}178.6 \pm 4.6 \\
(177.4 \pm 0.7)\end{array}$ & $15.78 \pm 0.05$ \\
\hline Jan. 15.0780 & NOT(+ALFOSC) & $12 \times 600$ & 0.9 & $0.85 \pm 0.28$ & $106.1 \pm 9.4$ & $18.28 \pm 0.05$ & - & - & _ \\
\hline Jan. 17.2518 & VLT(+FORS1) & $8 \times 285$ & 1.0 & $\begin{array}{c}0.84 \pm 0.45 \\
(1.14 \pm 0.51)\end{array}$ & $\begin{array}{c}138.3 \pm 8.1 \\
(141.0 \pm 7.2)\end{array}$ & $17.90 \pm 0.05$ & $\begin{array}{c}1.43 \pm 0.14 \\
(1.59 \pm 0.04)\end{array}$ & $\begin{array}{c}182.5 \pm 5.1 \\
(177.4 \pm 0.7)\end{array}$ & $15.69 \pm 0.05$ \\
\hline Jan. 30.1858 & VLT(+FORS1) & $4 \times 285$ & 0.8 & $\begin{array}{c}1.05 \pm 0.06 \\
(1.11 \pm 0.08)\end{array}$ & $\begin{array}{c}135.3 \pm 1.7 \\
(134.0 \pm 2.0)\end{array}$ & $17.31 \pm 0.05$ & $\begin{array}{c}1.64 \pm 0.06 \\
(1.59 \pm 0.04)\end{array}$ & $\begin{array}{c}178.5 \pm 1.1 \\
(177.4 \pm 0.7)\end{array}$ & $16.39 \pm 0.05$ \\
\hline Jan. 30.2022 & VLT(+FORS1) & $4 \times 285$ & 0.8 & $\begin{array}{c}1.28 \pm 0.06 \\
(1.21 \pm 0.08)\end{array}$ & $\begin{array}{c}132.5 \pm 1.4 \\
(133.3 \pm 1.9)\end{array}$ & $17.27 \pm 0.05$ & $\begin{array}{c}1.56 \pm 0.06 \\
(1.59 \pm 0.04)\end{array}$ & $\begin{array}{r}176.1 \pm 1.1 \\
(177.4 \pm 0.7)\end{array}$ & $16.36 \pm 0.05$ \\
\hline Feb. 11.9326 & CAHA(+CAFOS) & $13 \times 400$ & 2.0 & $\begin{array}{c}1.58 \pm 0.97 \\
(1.19 \pm 1.51)\end{array}$ & $\begin{array}{c}126.6 \pm 16.4 \\
(131.6 \pm 20.5)\end{array}$ & $17.82 \pm 0.09$ & $\begin{array}{c}0.92 \pm 0.88 \\
(1.59 \pm 0.04)\end{array}$ & $\begin{array}{r}179.8 \pm 26.0 \\
(177.4 \pm 0.7)\end{array}$ & $17.09 \pm 0.11$ \\
\hline Feb. 26.1107 & CAHA(+CAFOS) & $16 \times 600$ & 1.9 & $\begin{array}{c}<2.76^{f} \\
\left(<2.94^{f}\right)\end{array}$ & $\begin{array}{l}- \\
-\end{array}$ & $18.52 \pm 0.09$ & $\begin{array}{c}1.81 \pm 0.63 \\
(1.59 \pm 0.04)\end{array}$ & $\begin{array}{c}173.5 \pm 14.4 \\
(177.4 \pm 0.7)\end{array}$ & $17.56 \pm 0.08$ \\
\hline Feb. 26.9444 & NOT(+ALFOSC) & $16 \times 400$ & 1.3 & $0.98 \pm 0.80$ & $124.6 \pm 23.2$ & $18.79 \pm 0.08$ & - & - & - \\
\hline Mar. 2.1102 & VLT(+FORS1) & $4 \times 525$ & 1.1 & $\begin{array}{c}1.42 \pm 0.46 \\
(1.17 \pm 0.51)\end{array}$ & $\begin{array}{c}139.0 \pm 9.1 \\
(134.7 \pm 11.4)\end{array}$ & $18.80 \pm 0.05$ & $\begin{array}{c}1.83 \pm 0.19 \\
(1.59 \pm 0.04)\end{array}$ & $\begin{array}{c}174.6 \pm 3.2 \\
(177.4 \pm 0.7)\end{array}$ & $17.55 \pm 0.05$ \\
\hline Mar. 28.0207 & VLT(+FORS1) & $4 \times 525$ & 1.1 & $\begin{array}{c}0.75 \pm 0.57 \\
(0.73 \pm 0.78) \\
\end{array}$ & $\begin{array}{r}111.2 \pm 21.9 \\
(112.7 \pm 21.0)\end{array}$ & $19.42 \pm 0.05$ & $\begin{array}{c}1.58 \pm 0.42 \\
(1.59 \pm 0.04)\end{array}$ & $\begin{array}{r}179.6 \pm 7.6 \\
(177.4 \pm 0.7)\end{array}$ & $17.98 \pm 0.05$ \\
\hline
\end{tabular}

Notes. ${ }^{(a)}$ Mean observing epoch. ${ }^{(b)}$ For the NOT epochs SN 2007uy was out of the FoV, so the degree of linear polarization $(P)$, the position angle $(\theta)$, and the magnitude $(V)$ could not be determined. ${ }^{(c)}$ Calibration based on Malesani et al. (2009). The photometric errors include the zero point uncertainty. ${ }^{(d)}$ The polarimetric cycle was interrupted and restarted several times. The SN 2007uy observing epoch and the exposure time are slightly different from the SN 2008D/XT 080109 ones, corresponding to Jan. 13.2708 UT and 990 seconds, respectively. ${ }^{(e)}$ The SN 2008D/XT $080109 P$ and $\theta$ values given for VLT and CAHA in between parentheses assume a constant SN 2007uy polarization given by $\langle P\rangle=1.59 \pm 0.04 \%$ and $\langle\theta\rangle=177.4 \pm 0.7^{\circ}{ }^{(f)} 1 \sigma$ upper limit.

combining sky flat field frames taken without polarization units in the light path. This process was applied separately for the 3 instruments. In principle, the host galaxy background might pose a potential problem for the photometric accuracy of SN 2007uy and SN 2008D/XT 080109. Inspection of the NGC 2770 region around each SN shows that SN 2007uy is surrounded by a more inhomogeneous background than SN 2008D/XT 080109. Thus we double-checked the impact of the host galaxy background level determination by performing aperture photometry with radii ranging from 0.5 to 2.5 times the $F W H M$ (Full Width at Half Maximum), and varying the inner radii of the background annuli from 3 to 5 times the FWHM. The annuli widths were also varied from 2.5 to 4 times the $F W H M$. We verified that the resulting Stokes parametres were consistent within error bars, independent of the apertures and annuli used. Then the apertures and annuli yielding minimum errors were adopted (typically the apertures and the inner radii used were the FWHM and 3-4 times the $F W H M$, respectively). In any case, in order to account for potentially dismissed photometric error sources, the statistical analysis of Sect. 3.2.1 was duplicated considering also photometric errors augmented by $20 \%$.

For the ALFOSC and CAFOS data the determination of the Stokes parametres was done by fitting the $S(\theta)$ function with the corresponding Milky Way GIP normalization factor (di Serego Alighieri 1997). The GIP normalization factor was calculated using Galactic field stars.

In the case of FORS1 the Stokes parametres were calculated via Fourier expansion (see expressions 4.5 and 4.6 of FORS1 manual $^{2}$ ). Then the Stokes parametres of all the objects in the images were corrected for the FORS1 spurious polarization

Astronomy (AURA) under cooperative agreement with the National Science Foundation.

2 Doc. N. VLT-MAN-ESO-13100-1543. Issue 82.1, date 27/02/2008. correction, which considers the instrumental polarization dependence across the $\mathrm{FoV}^{3}$. Next, the $V$-band angle offset of the $1 / 2$ wave retarder plate was included (given in the Table 4.7 of FORS1 manual). All the objects (including both $\mathrm{SNe}$ ) of all FORS1 visits used the same angle offset, minimizing a potential source of internal scattering in the Stokes plane. Finally, the GIP correction was introduced by shifting the origin of the Stokes plane to the barycentre of the field stars ${ }^{4}$.

Due to the reduced ALFOSC FoV only two unsaturated bright field stars were available for the GIP correction of the NOT data. Fortunately for the three NOT epochs the stars used for the NOT GIP correction remained the same. This assures internal consistency of the position of the NOT Stokes parametres on the Stokes plane. Thus, a potential relative shift (owing to different GIP corrections) of the NOT Stokes parametres with respect to each other was minimized. This fact will be of relevance in the further discussion since, as will be argued in Sect. 3.2.2, the polarimetric data might suggest the existence of a symmetry axis which is consistently determined by different instrumental setups.

The wider FoV of FORS1 and CAFOS provided a larger number of stars for the GIP correction than with ALFOSC. Due to different observing conditions (seeing, transparency and CCD binning) at VLT and CAHA the stars varied from one night to another. This was not critical for the FORS1 and CAFOS images, since the GIP correction for both SN 2008D/XT080109 and SN 2007uy were always calculated using the same set of stars for each epoch. And most importantly, given that SN 2007uy was very well detected in all the VLT and CAHA images, we could use this object (justified by a statistical analysis, see Sect. 3.2.1)

\footnotetext{
3 http://wWw.eso.org/paranal/instruments/fors1/inst/ pola.html

${ }_{4}^{4}$ The FORS1 reduction steps explained above follow the recommendations given by Dr. T. Szeifert, priv. comm.
} 
as a secondary calibrator and keep it fixed on the Stokes plane (see Sect. 3.2.2).

It is important to stress that the GIP corrections applied to the different data-sets were very similar since the Galactic extinction towards NGC 2770 is only $A_{V}=0.1 \mathrm{mag}$ (Schlegel et al. 1998; Cardelli et al. 1989). So in all cases the GIP corrections applied were low $(\sim 0.2-0.3 \%)$, in agreement with the Galactic polarization predictions $\left(P_{\max }=2.9 \% \times A_{V}=0.29 \%\right.$; (Serkowski et al. 1975; Cardelli et al. 1989). In fact, the GIP corrections were always well below the typical level of our polarization detections $(\sim 1-1.5 \%)$.

Verification of the photometry, calibration and the reduction was done by observation of polarimetric standards. The standard stars observed were NGC 2024-1 and Vela1-95 at VLT (Fossati et al. 2007; Whittet et al. 1992), HD 19820 at CAHA (Wolff et al. 1996), and HD 94851, BD 25727, HD 251204 and G191B2B at NOT (Turnshek et al. 1990; Schmidt et al. 1992). Table 1 shows the inferred $\theta$ and $P$ values, once they were corrected for the GIP and the statistical bias (multiplying $P$ by $\sqrt{1-\left(\sigma_{P} / P\right)^{2}}$ ) due to the fact that $P$ is a positive quantity (Wardle \& Kronberg 1974). The SN 2008D/XT $080109 P$ and $\theta$ values displayed in Table 1 for VLT and CAHA in between parentheses assume a constant SN 2007uy polarization given by $\langle P\rangle=1.59 \pm 0.04 \%$ and $\langle\theta\rangle=177.4 \pm 0.7^{\circ}$.

For some observing epochs we explored maximising the time resolution of our polarimetric monitoring at the expense of enlarging the polarimetric errors. This was done, when possible, by splitting the polarimetric data of one night in cycles of four images, i.e., the minimum block of images necessary to get a polarimetric point. Unfortunately most of the polarization detections showed modest significance levels (between $1.1 \sigma$ and $4.7 \sigma$ ), so we could not split the data. Only the VLT dataset of January 30 could be divided in two cycles of four images, while still keeping high-significance polarization detections (above $17.5 \sigma$ for each of the two cycles). We verified that the joint data acquired on January 30 (composed of two cycles of four images) yields Stokes parametres consistent with the ones obtained when the data of that night are split in two halves. This separation provided an extra high-quality data-point for the statistical analyses carried out in Sects. 3.2.1-3.2.3.

The $V$-band polarimetry values synthesized by Maund et al. (2009) for SN 2008D/XT 080109 on January 31.22 and February 15.18 UT bring us the opportunity to cross-check our results. Our two closest observations, carried out on January 30.2022 and February $11.9326 \mathrm{UT}$, yield $P$ and $\theta$ values fully consistent with Maund et al. (2009). We are aware that this comparative exercise, although satisfactory, is only limited to two epochs close to the light curve maximum and exclusively focused on SN 2008D/XT 080109, since Maund et al. (2009) did not observe SN 2007uy.

\subsection{Millimetre observations}

SN 2008D/XT 080109 was observed at $1.2 \mathrm{~mm}$ on January 25, 28, and 30, 2008 with the MAMBO II bolometer on the IRAM $30 \mathrm{~m}$ Telescope (see Table 2). The data were reduced following the standard procedure with the mopsic data reduction software. In order to estimate the NGC 2770 contribution at $1.2 \mathrm{~mm}$ to our previous SN 2008D/XT080109 observations we measured, on January 31, the flux of two adjacent regions (Adj1 and Adj2). Such regions bracket the SN2008D/XT080109 position in the radial direction towards the NGC $2770 \mathrm{nu}-$ cleus, both at a distance of $\sim 10^{\prime \prime}$ from SN 2008D/XT 080109
Table 2. Log of the millimetre observations.

\begin{tabular}{cccccc}
\hline \hline $\begin{array}{c}\text { Date } \\
\text { 2008, UT }\end{array}$ & Telescope & Field & \multicolumn{2}{c}{$\begin{array}{c}\text { Integration } \\
\text { time }(\mathrm{min})\end{array}$} & $\begin{array}{c}\text { Flux density } \\
\text { [mJy/beam }]\end{array}$ \\
\hline Jan. 25.931 & IRAM 30 m SN 2008D & $3 \times 20$ & 1.2 & $2.46 \pm 0.54$ \\
Jan. 28.200 & IRAM 30 m SN 2008D & $1 \times 20$ & 1.2 & $2.29 \pm 1.20$ \\
Jan. 30.171 & IRAM 30 m SN 2008D & $2 \times 20$ & 1.2 & $1.44 \pm 0.60$ \\
Jan. 25.931-30.171 & IRAM 30 m SN 2008D & $6 \times 20$ & 1.2 & $2.03 \pm 0.38^{b}$ \\
Jan. 31.925 & IRAM 30 m & Adj1 $^{c}$ & $2 \times 16$ & 1.2 & $2.39 \pm 0.70$ \\
Jan. 31.936 & IRAM 30 m & Adj2 $^{d}$ & $2 \times 16$ & 1.2 & $2.81 \pm 0.71$ \\
\hline Jan. 23.854 & PdB & SN 2008D & $1 \times 60$ & 3.3 & $0.65 \pm 0.15$ \\
Nov. 9.336 & PdB & SN 2008D & $1 \times 111$ & 2.9 & $0.03 \pm 0.10$ \\
\hline
\end{tabular}

Notes. ${ }^{(a)}$ Mean observing epoch. ${ }^{(b)}$ Weighted average of the three previous table lines. ${ }^{(c)}$ RA $=09^{\mathrm{h}} 09^{\mathrm{m}} 30^{\mathrm{s}} 085$, Dec. $=+33^{\circ} 08^{\prime} 29^{\prime} .98$ $(\mathrm{J} 2000) .{ }^{(d)} \mathrm{RA}=09^{\mathrm{h}} 09^{\mathrm{m}} 31^{\mathrm{s}} .211$, Dec. $=+33^{\circ} 08^{\prime} 10^{\prime} .25(\mathrm{~J} 2000)$.

(beam $F W H M$ at $1.2 \mathrm{~mm} \sim 11^{\prime \prime}$ ). They were selected sufficiently close to SN 2008D/XT 080109 to have a good estimate of its background emission, but sufficiently far to avoid most of the SN 2008D/XT 080109 flux within the beam. The adjacent $1.2 \mathrm{~mm}$ pointings yielded fluxes consistent with those obtained at the SN 2008D/XT080109 position (see Table 2), implying that the $1.2 \mathrm{~mm}$ data are very likely dominated by the NGC 2770 background flux and not by SN 2008D/XT 080109.

In addition, a $4.3 \sigma$ detection was achieved on January 23 at $3.3 \mathrm{~mm}$ with the Plateau de Bure (PdB) interferometer centered on SN 2008D/XT 080109 (see Table 2) with an observing beam size of 2 '. $75 \times 1$ '. 11 . The last millimetre observation was carried out on November $9.336 \mathrm{UT}$ with $\mathrm{PdB}$ at $2.9 \mathrm{~mm}$, yielding a $3 \sigma$ upper limit of $0.30 \mathrm{mJy} / \mathrm{beam}$. This non-detection suggests that the flux detected at $3.3 \mathrm{~mm}$ on January 23 mostly originated from SN 2008D/XT 080109 and not from the host galaxy dust.

\section{Results}

\subsection{Properties of the polarizing ISM towards SN2008D/XT 080109}

The Galactic reddening towards NGC 2770 is very low (Schlegel et al. 1998, $E(B-V)=0.032)$, implying a $V$-band extinction of only $A_{V}=0.1 \mathrm{mag}$ (Cardelli et al. 1989). Therefore the contribution of the Galactic dust to the measured $1.2 \mathrm{~mm}$ flux is negligible. Thus, assuming that most of the $1.2 \mathrm{~mm}$ emission comes from optically thin dust emission in the host galaxy interstellar medium (ISM) towards SN 2008D/XT 080109, it is possible to roughly estimate the host optical extinction as: $A_{V}=$ $\left(1.086 S_{1.2}\right) /\left(B_{1.2}\left(T_{\text {dust }}\right) \Omega_{\mathrm{mb}}\right) \times\left(\kappa_{V} / \kappa_{1.2}\right) . B_{1.2}\left(T_{\text {dust }}\right)$ is the Planck function of the dust at a temperature $T_{\text {dust }}, \Omega_{\mathrm{mb}}$ is the mainbeam solid angle, $S_{1.2}$ is the flux density per beam at $1.2 \mathrm{~mm}$, and $\kappa_{V} / \kappa_{1.2}$ is the ratio of the visual extinction coefficient to the $1.2 \mathrm{~mm}$ dust opacity, which was estimated to be $\left\langle\kappa_{V} / \kappa_{1.2}\right\rangle=$ $(4 \pm 2) \times 10^{4}$ (Kramer et al. 1998).

In order to estimate $A_{V}$ a value of $T_{\text {dust }}$ has to be assumed. Domingue et al. (1999) inferred $T_{\text {dust }}=21 \pm 2 \mathrm{~K}$ for the colder dust component of a set of three spiral galaxies similar to NGC 2770. On the other hand, the spectral energy distribution of NGC 2770 integrated over the entire galaxy yields $T_{\text {dust }}=30 \pm 5 \mathrm{~K}$ (Thöne et al. 2009). So we adopted both $T_{\text {dust }}=21 \pm 2 \mathrm{~K}$ and $T_{\text {dust }}=30 \pm 5 \mathrm{~K}$.

Using $S_{1.2}=(2.03 \pm 0.38) \mathrm{mJy} /$ beam (line four of Table 2 ; weighted average of the first three table lines) and both $T_{\text {dust }}=$ $21 \pm 2 \mathrm{~K}$ and $T_{\text {dust }}=30 \pm 5 \mathrm{~K}$, we obtain $A_{V}=0.43 \pm 0.31$ 
and $A_{V}=0.27 \pm 0.15$, respectively. However, as $S_{1.2}$ corresponds to the flux integrated in the beam along the projected thickness of the host galaxy (which includes contribution from any ISM located beyond the XT), we can only provide an upper limit to $A_{V}$. To be conservative, we will assume an extinction of $A_{V}<0.43+0.31=0.74$, obtained with $T_{\text {dust }}=21 \pm 2 \mathrm{~K}$. This $A_{V}$ value is $\sim 0.5-1.8$ mag lower than the line-of-sight $A_{V}$ values inferred by other authors based on a broad diversity of techniques (Mazzali et al. 2008; Soderberg et al. 2008; Malesani et al. 2009; Modjaz et al. 2009). An unrealistic temperature of $T_{\text {dust }} \sim 10 \mathrm{~K}$ would be necessary to have an $A_{V}$ value in agreement with the above authors.

This apparent disagreement can be explained by the impossibility to resolve a clumpy host galaxy ISM with our $1.2 \mathrm{~mm}$ beam $\left(F W H M=11^{\prime \prime}\right)$. The clumpy ISM would show three main properties; i) an ISM composed of cells superimposed on a lower extinction background; ii) cells displaying typical angular sizes smaller than the beam size; iii) typical angular separation between cells small enough to allow the beam to contain several cells. Given that the extinction determined by the $1.2 \mathrm{~mm}$ data is an average of the integrated flux received in the beam, this scenario would explain the higher extinction derived along the XT line-of-sight (i.e., spectroscopy). On the other hand, condition iii) would explain the fact that the pointings adjacent to the SN 2008D/XT 080109 position yielded similar $1.2 \mathrm{~mm}$ fluxes, and hence extinction values.

We can estimate a rough upper limit of the typical cell sizes as follows. First, we assume that the flux differences (or internal dispersion) between our three $1.2 \mathrm{~mm}$ pointings (see Table 2; weighted flux average of SN 2008D/XT080109, Adj1 and Adj2) were entirely due to the statistical fluctuations in the average number of cells $(N)$ contained in each beam. In our case the three $1.2 \mathrm{~mm}$ pointings yield $2.03 \pm 0.38$, $2.39 \pm 0.70$ and $2.81 \pm 0.71 \mathrm{mJy} /$ beam, so the dispersion is $0.41 \mathrm{mJy} / \mathrm{beam}$. If the cells were identical and randomly distributed, then the ratio between the average flux and the dispersion of the 3 pointings would be approximately $\sqrt{N}$. In our case the average flux of the 3 pointings (calculated by weighting with the corresponding flux errors) is $2.24 \mathrm{mJy} / \mathrm{beam}$, so this yields $N \sim 30$ cells/beam. However, the dispersion is very likely not only due to statistical fluctuations of $N$, i.e., the dispersion probably also has an instrumental/calibration/photon-noise component. So the statistical fluctuations due to $N$ would be likely lower than $0.41 \mathrm{mJy} /$ beam and therefore we can only set a lower limit of $N>30$.

Additionally, in order to match the $A_{V}$ derived from the $1.2 \mathrm{~mm}$ flux with the spectroscopic $A_{V}$ values reported in the literature, the emitting region (the total area covered by the cells within the beam solid angle) should fill only ${ }^{5} 10-30 \%$ of $\Omega_{\mathrm{mb}}$. This would explain the low $A_{V}<0.74$ mag derived using the $1.2 \mathrm{~mm}$ flux (Kramer et al. 1998) for cells having a line-of-sight spectroscopic extinction of $A_{V}=1.2-2.5 \mathrm{mag}$. Thus, assuming identical circular cells, a NGC 2770 distance of $27 \mathrm{Mpc}$ (Soderberg et al. 2008), and a $\Omega_{\mathrm{mb}}$ filling factor of $30 \%$, we can impose a maximum cell diameter of $D<0.16 \mathrm{kpc}$. Adopting a beam filling factor of $10 \%$, we would obtain smaller cells, so we consider $0.16 \mathrm{kpc}$ as a robust upper limit. As a comparison it is interesting to mention that most ( $98 \%)$ of the giant molecular clouds resolved by millimetric observations in the nearby spiral M 33 show sizes between 0.05 and $0.16 \mathrm{kpc}$ (Rosolowsky et al. 2007), compatible with our observations. Similarly, most of the HII regions in nearby spiral galaxies show physical scales

${ }^{5}$ For dust temperatures ranging from $T=21$ to $T=30 \mathrm{~K}$. consistent with <0.16 kpc (González-Delgado \& Pérez 1997). We note that the dominant $P$ is the result of all the material integrated along the line-of-sight, so we can not conclude that the dominant $P$ is exclusively due to the XT circumstellar dust.

\subsection{Properties of SN2008D/XT080109 and SN2007uy on the Stokes plane}

The $P$ and $\theta$ values displayed in Table 1 were corrected for the GIP and the polarimetric statistical bias (Wardle \& Kronberg 1974). The further polarimetric analysis will deal with the properties of $Q$ and $U$ of both objects on the Stokes plane, so the $P$ bias correction factor (included in Table 1) will not be considered.

\subsubsection{Is SN $2008 \mathrm{D} / \mathrm{XT} 080109$ polarimetrically variable?}

The Stokes parametres of SN2008D/XT080109 and SN 2007uy, corrected for the GIP, are plotted in Fig. 1. Both events show polarization levels of $1-1.5 \%$, significantly lower than the one measured in XRF 060218/SN2006aj (Gorosabel et al. 2006; Maund et al. 2007). Assuming both a GIP law and a Galactic extinction law (Serkowski et al. 1975; Cardelli et al. 1989) for NGC 2770, the $A_{V}$ values reported in the literature ( 1.2-2.5 mag) would yield a conservative HGIP polarization upper limit of $P_{\max }=2.9 \% \times A_{V} \sim 3.5-7.3 \%$, consistent with the polarization measured for SN 2008D/XT 080109 and SN 2007uy.

As seen in the upper panel of Fig. 1, the cloud of points corresponding to SN2007uy seems to be more clustered than the one of SN 2008D/XT 080109. For the two stellar explosions we quantified the probability that the Stokes parametre distributions are consistent with no time evolution. First, we double-checked using Monte Carlo methods that, for the average number of counts of our images, both $Q$ and $U$ follow Gaussian distributions. This fact assures that the $\chi^{2}$ test is an appropriate statistical tool. Second, we determined the barycentre of the two objects on the Stokes plane $(\langle Q\rangle,\langle U\rangle$; see the long-dashed lines of Fig. 2). Then we calculated for the $Q$ and $U$ of the two events the $\chi^{2} /$ d.o.f. value with respect to $\langle Q\rangle$ and $\langle U\rangle$, respectively ${ }^{6}$.

For SN 2007uy the distribution of $Q(U)$ shows $\chi^{2} /$ d.o.f. $=$ $4.18 / 7\left(\chi^{2} /\right.$ d.o.f. $\left.=4.32 / 7\right)$ with respect to $\langle Q\rangle(\langle U\rangle)$. For SN 2008D/XT080109 the distribution of $Q(U)$ yields $\chi^{2} /$ d.o.f. $=19.06 / 10\left(\chi^{2} /\right.$ d.o.f. $\left.=22.96 / 10\right)$, clearly larger than the $\chi^{2} /$ d.o.f. value of SN 2007uy. These values of $\chi^{2} /$ d.o.f. were used to obtain the corresponding probabilities. For SN 2007uy the probability that $Q$ and $U$ are constant are 0.758 and 0.742 , respectively. SN 2008D/XT 080109 shows lower probabilities, 0.040 and 0.011 , respectively. Thus the probabilities that $Q$ and $U$ are simultaneously constant are 0.563 for SN 2007uy and $4.3 \times 10^{-4}$ for SN $2008 \mathrm{D} / \mathrm{XT} 080109$.

We are aware that different error sources affecting the measurements (background determination uncertainties, flat field correction inaccuracies, no instrumental polarization correction available along the FoV for the CAHA and NOT images, etc.) might still not be included in the error bars, so the values of $\chi^{2} /$ d.o.f. could be overestimated for both sources (and hence the above probabilities underestimated). Thus, we repeated the exercise augmenting the photometric error bars of both objects and all the field stars by $20 \%$. If we do that, the probability that

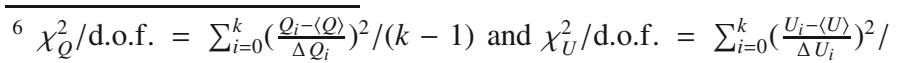
$(k-1), k$ being the number of visits and d.o.f. $=k-1$ the degrees of freedom.
} 


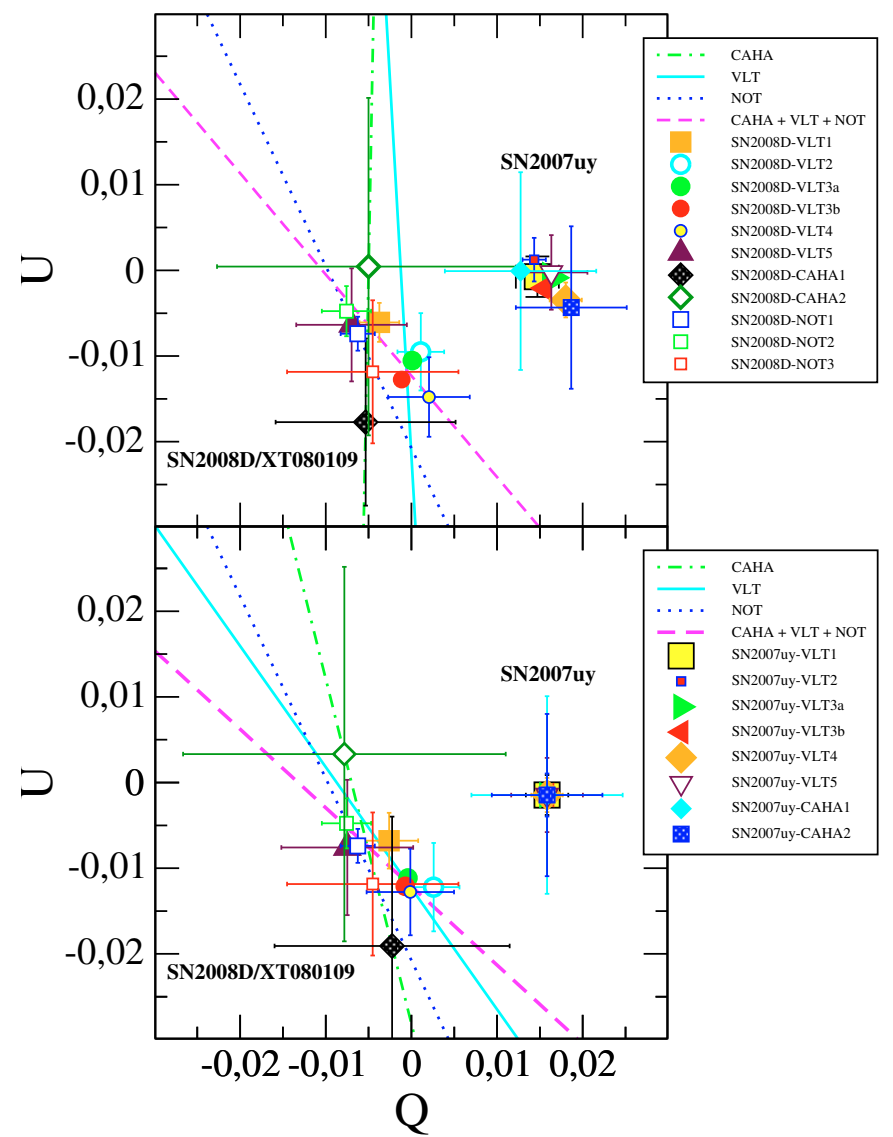

Fig. 1. Upper panel: Stokes parametres of SN 2008D/XT 080109 and SN 2007uy, once the GIP correction has been included. The cloud of measurements representing SN 2007uy seems to be more compact than the SN 2008D/XT 080109 one. The different lines represent the linear fits to the SN 2008D/XT 080109 Stokes parametres obtained when the CAHA (dot-dashed), VLT (continuous), NOT (dotted) and all the data (VLT+CAHA+NOT, long-dashed) are considered. The directions of all the straight lines are statistically consistent (see Table 4), which might indicate the presence of a preferential axis. This possible evidence is strengthened when SN2007uy is used as reference (see below). Bottom panel: Stokes parametres of SN 2008D/XT 080109 and SN 2007uy, if SN2007uy is kept fixed at its barycentre. The directions of all the straight lines are statistically more consistent than in the upper panel (compare the internal consistency of the different orientations in Tables 3 and 4), reinforcing the possible presence of an instrumental-independent dominant symmetry axis present in the SN 2008D/XT 080109 data.

both $Q$ and $U$ are constant are 0.791 for SN 2007uy and 0.021 for SN 2008D/XT 080109, respectively.

We have checked, if due to some uncontrolled reason, the $(Q, U)$ distribution on the Stokes plane is just the result of mixing data acquired with different telescopes/instruments. In order to inspect the existence of this potential instrumental artifact, we redid the statistical analysis separating the images that contain both objects according to the telescope used (hence the NOT is excluded from this comparative study between SN 2007uy and SN 2008D/XT 080109 since SN 2007uy was not imaged by the NOT). Thus we determined separately for the VLT and CAHA the probabilities of having constant Stokes parametres.

Using only the VLT data points, we determined that the probability that both $Q$ and $U$ are constant are 0.295 for SN 2007uy and 0.007 for SN 2008D/XT 080109, respectively. If we enlarge the photometric errors by $20 \%$ the probabilities would be then 0.532 and 0.056, for SN 2007uy and SN 2008D/XT 080109, respectively. Considering exclusively the CAHA data-set we can not reach strong conclusions given the reduced number of visits (2) and large error bars. However, even with the limited CAHA data, SN 2007uy shows a higher probability than SN 2008D/XT 080109 of being polarimetrically constant, 0.457 versus 0.403 . If the CAHA photometric errors are augmented by $20 \%$, then the probabilities are 0.530 and 0.423 for SN 2007uy and SN 2008D/XT 080109, respectively.

Independent of the data subset considered and the additional photometric uncertainty introduced, SN 2008D/XT 080109 reiteratively displays lower probabilities than SN 2007uy of being polarimetrically constant. In fact, it is not obvious to explain how non-accounted photometric/calibration/instrumental uncertainties could systematically affect only the values derived for SN 2008D/XT 080109 and not those of SN 2007uy, which consistently shows a higher degree of clustering on the Stokes plane. It is interesting to note that this is against the a priori expectations, given that the more inhomogeneous host background of SN 2007uy (in comparison to SN 2008D/XT 080109) might represent a potential uncontrolled source of noise in its Stokes parametres. All the above arguments seem to suggest that SN 2008D/XT 080109 could show an intrinsic variable polarization component added to the dominant HGIP. This suggestion is reinforced when SN 2007uy is used as a secondary polarimetric standard (see Sect. 3.2.2). However, it is important to note that a constant polarization signal from SN 2007uy is atypical of previously observed core-collapse SNe (Wang \& Wheeler 2008). We point out that none of the two SNe seem to obey any obvious smooth long-term polarimetric evolution correlated to their light curves (see Fig. 2).

\subsubsection{A symmetry axis for SN 2008D/XT 080109?}

Given that both SN 2007uy and SN 2008D/XT080109 were imaged simultaneously with VLT and CAHA, we can use SN 2007uy as a reference star with constant Stokes parametres. In other words, we can assume, justified by the above probabilities, a constant projected geometry for SN 2007uy plus a constant HGIP. As an additional argument, it is also interesting to note that the agreement between the synthetic broad-band points reported by Maund et al. (2009) and our data is even better when SN 2007uy is fixed on the Stokes plane.

Thus, using SN2007uy as calibrator, a linear fit to the SN 2008D/XT 080109 data yields a satisfactory $\chi^{2} /$ d.o.f. = 1.9/9 (long-dashed straight line of Fig. 1 bottom panel). Hence, the SN 2008D/XT 080109 Stokes parametres might suggest the existence of a dominant symmetry axis, offset from the origin due to the HGIP. Furthermore, as seen in the first three lines of Table 3 the inferred symmetry axes show a preference to negative orientations independent of the telescope+instrument employed in the observations 7 . This effect can be seen in the bottom panel of Fig. 1, which shows the linear fits carried out both when the data are considered separately (CAHA, VLT, NOT) and jointly (CAHA+VLT+NOT). The linear fits were obtained minimizing $\chi^{2}$, which is defined as the weighted distance perpendicular to the fitted line ${ }^{8}$. We considered this $\chi^{2}$ definition because the variables to be fitted $(Q, U)$ and their corresponding

\footnotetext{
7 We define the orientation as the angle of the fitted straight line with respect to the horizontal axis representing $Q$ on the Stokes plane. The orientation ranges between -90 and +90 degrees.

8 Also known as weighted total least squares (WTLS), weighted rigorous least squares, or weighted orthogonal regression method (see Lemmerling \& van Huffel 2002, and references therein).
} 


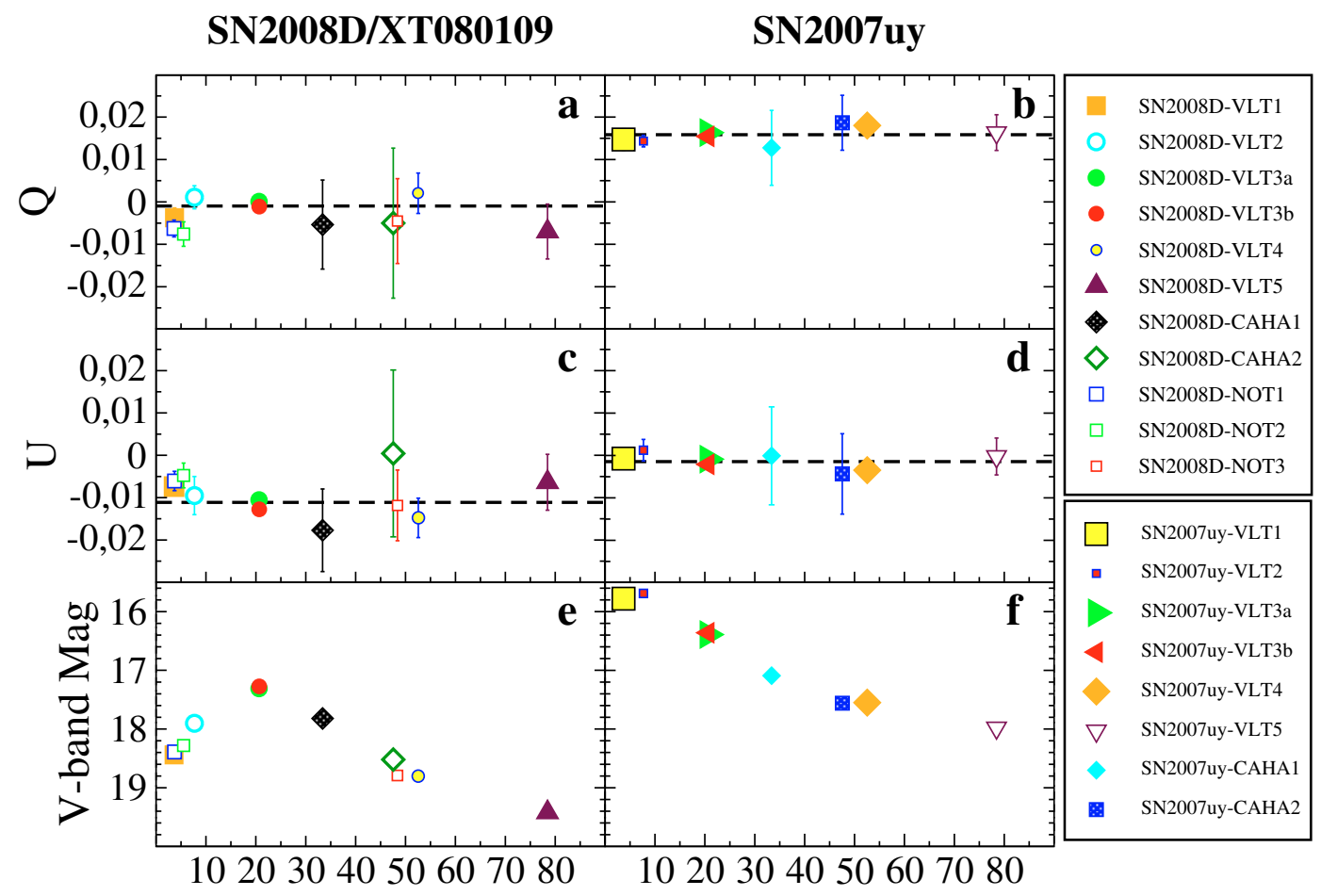

\section{Days from SN2008D X-ray outburst}

Fig. 2. Polarimetric and photometric evolution of SN 2008D/XT 080109 (panels a), c), e)) and SN 2007uy (panels b), d), f)). For consistency we used the same symbols as in Fig. 1. The long-dashed lines indicate the position of the barycentre $(\langle Q\rangle,\langle U\rangle)$ of each SN. The two bottom panels e) and f) show the $V$-band light curves of SN 2008D/XT 080109 and SN 2007uy uncorrected for Galactic reddening. None of the two SNe seem to obey any obvious smooth long-term polarimetric evolution correlated with their optical light curves.

errors are treated symmetrically (Boogs et al. 1990; Babu \& Feigelson 1996).

In order to quantify the degree of consistency that the symmetry axes of the 3 data-sets could show (when constant SN 2007uy Stokes parametres are assumed) we mapped the evolution of the linear fit $\chi^{2} /$ d.o.f. when the orientation of the symmetry axis is varied from -90 to +90 degrees. The upper panel of Fig. 3 shows this evolution. We warn the reader that in the particular case of CAHA d.o.f. $=0$, so what is plotted for CAHA in Fig. 3 upper panel actually represents $\chi^{2}$. As seen all the three independent data-sets show their $\chi^{2} /$ d.o.f. minima approximately at the same orientation. The position of all the $\chi^{2} /$ d.o.f. minima were positively confirmed using the fi texy routine implemented by Press et al. (1992). The lower panel of Fig. 3 shows the normalized probabilities corresponding to the $\chi^{2} /$ d.o.f. values of the above panel.

For the case of CAHA, given that d.o.f. $=0$, the plotted probability evolution was obtained by simulating mock CAHA Stokes parametres created by a weighted Monte Carlo method assuming Gaussian errors for $Q$ and $U$. Then given two simulated $(Q, U)$ CAHA pairs the orientation of the line passing through those Stokes parametres is determined. The repetition of this process allowed us to construct the histogram of the line orientations for the CAHA data. Thus, the lower panel of Fig. 3 displays for CAHA the histogram of orientations for $10^{7}$ Monte Carlo simulations?.

9 This Monte Carlo method, used for determining the probability distribution for CAHA, is not optimal for the VLT and NOT data. For the VLT and the NOT a set of simulated Stokes parametres does not determine a line passing through them (they contain more than 2 visits) and a formal fit is necessary. So, the histogram of orientations would mix linear fits with different qualities.

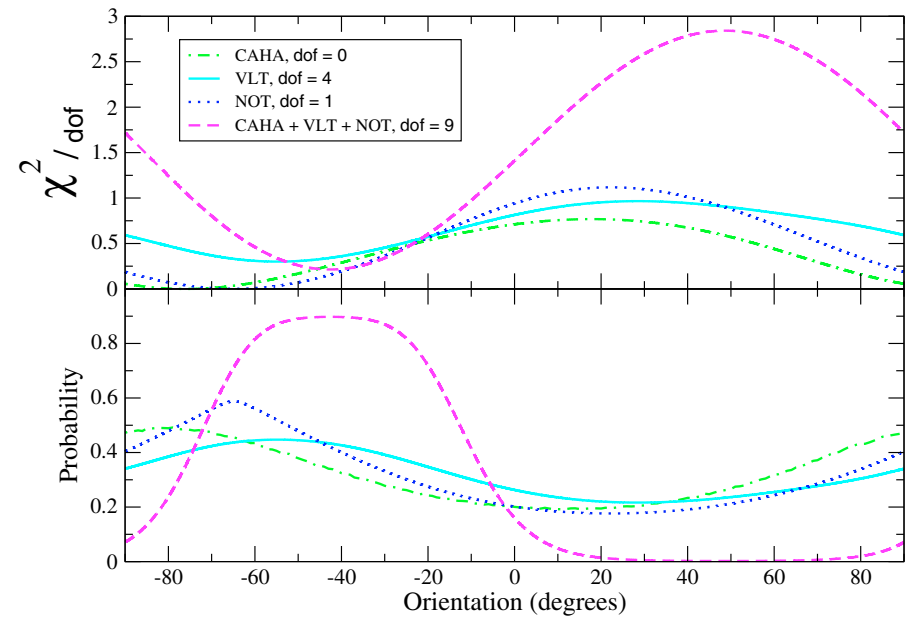

Fig. 3. Upper panel: the evolution of the linear fit $\chi^{2} /$ d.o.f. as a function of the fitted line orientation when constant polarization is assumed for SN 2007uy. The minima correspond to the optimal orientation of the straight lines displayed in Fig. 1 bottom panel, also given in Table 3. As seen the global shape of the evolution for the three independent datasets (VLT, NOT and CAHA) are quite equivalent, their minima being systematically located at similar negative angles. The long-dashed line shows the $\chi^{2} /$ d.o.f. evolution when all the data are jointly considered. We note that in the case of CAHA, given that d.o.f. $=0, \chi^{2} /$ d.o.f. actually represents $\chi^{2}$. Bottom panel: the evolution of the normalized probability associated with the above panel. As displayed the three independent data-sets show their probability maxima at a comparable orientation angle. The maximum is reinforced when all the data are combined (long-dashed line). Given that d.o.f. $=0$ for CAHA, the corresponding probability distribution was obtained through a Monte Carlo method. We refer the reader to the main text for further information. 
Table 3. Linear fits on the Stokes plane of SN 2008D/XT 080109.

\begin{tabular}{lccccc}
\hline \hline Telescope & $\begin{array}{c}\text { Number of } \\
\text { visits }\end{array}$ & $\begin{array}{c}\text { Minimum } \\
\chi^{2} / \text { d.o.f. }\end{array}$ & $\begin{array}{c}\text { Orientation angle } \\
\text { (degrees) }\end{array}$ & $\begin{array}{c}\text { Lower error bar } \\
\text { (degrees) }\end{array}$ & $\begin{array}{c}\text { Upper error bar } \\
\text { (degrees) }\end{array}$ \\
\hline CAHA & 2 & $0^{a}$ & -76.1 & $49.4^{b}$ & $48.5^{b}$ \\
NOT & 3 & $0.001 / 1$ & -64.8 & 46.1 & 46.7 \\
VLT & 6 & $1.198 / 4$ & -54.8 & 52.2 & 51.8 \\
CAHA+VLT+NOT & 11 & $1.922 / 9$ & -42.6 & 23.3 & 22.9 \\
\hline
\end{tabular}

Notes. ${ }^{(a)}$ In this particular case, $\chi^{2}=0$ and d.o.f. $=0$ for the minimum, so $\chi^{2} /$ d.o.f. could also be formally represented by $0 / 0 .{ }^{(b)}$ Errors obtained through Monte Carlo methods.

The linear fits are shown in the bottom panel of Fig. 1, where we used SN 2007uy as a reference star with constant Stokes parametres. Column 1 displays the telescope used and Col. 2 the number of visits or points on the Stokes plane. Column 3 provides the $\chi^{2} /$ d.o.f. of the optimal linear fit. Column 4 shows the orientation angles at the minima (maxima) of Fig. 3 upper (bottom) panel. Columns 5 and 6 provide the lower and upper error bars around the orientation angle of the linear fit. As seen the three independent data-sets (CAHA, NOT and VLT) show orientations well consistent within errors. Last table line reports the results when all the data are combined.

Table 4. Linear fits on the Stokes plane of SN 2008D/XT 080109 when no constant Stokes parametres are assumed for SN 2007uy.

\begin{tabular}{lccccc}
\hline \hline Telescope & $\begin{array}{c}\text { Number of } \\
\text { visits }\end{array}$ & $\begin{array}{c}\text { Minimum } \\
\chi^{2} / \text { d.o.f. }\end{array}$ & $\begin{array}{c}\text { Orientation angle } \\
\text { (degrees) }\end{array}$ & $\begin{array}{c}\text { Lower error bar } \\
\text { (degrees) }\end{array}$ & $\begin{array}{c}\text { Upper error bar } \\
\text { (degrees) }\end{array}$ \\
\hline CAHA & 2 & $0^{a}$ & 88.9 & $53.9^{b}$ & $48.8^{b}$ \\
NOT & 3 & $0.001 / 1$ & -64.8 & 46.1 & 46.7 \\
VLT & 6 & $5.501 / 4$ & -86.7 & 27.9 & 26.8 \\
CAHA+VLT+NOT & 11 & $9.599 / 9$ & -49.8 & 17.4 & 17.2 \\
\hline
\end{tabular}

Notes. ${ }^{(a)}$ In this particular case, $\chi^{2}=0$ and d.o.f. $=0$ for the minimum, so $\chi^{2} /$ d.o.f. could also be formally represented by $0 / 0{ }^{(b)}$ Errors obtained through Monte Carlo methods.

The corresponding straight lines are shown in the upper panel of Fig. 1. Column 1 displays the telescope used and Col. 2 the number of visits or points on the Stokes plane. Column 3 provides the $\chi^{2} /$ d.o.f. of the optimal linear fit. Column 4 shows the orientation angles at the minima (maxima) of Fig. 3 upper (bottom) panel. Columns 5 and 6 provide the lower and upper error bars around the orientation angle of the linear fit. As seen the three independent data-sets (CAHA, NOT and VLT) show more scattered orientations (and also larger $\chi^{2} /$ d.o.f. values) than the ones displayed in Table 3, but they are still consistent within errors. Last table line reports the results when all the data are combined.

For each data-set the upper and lower error bars in the orientation angle (displayed in Table 3, Cols. 5 and 6) were calculated by integrating the $\pm 34.145 \%$ percentile of the probability distribution around the corresponding maximum. As seen in Table 3 the axes inferred from the three independent data-sets are consistent within errors. Hence, the inferred symmetry axes are statistically consistent in all cases, regardless of the instrumental setup used to determine it. It is also interesting to remark that the NOT Stokes parametres, which did not use SN 2007uy as a secondary calibrator (so they might apply a slightly different GIP correction), are also satisfactorily fitted by a straight line. Furthermore, the orientation of the NOT linear fit agrees with the ones inferred based on the VLT and CAHA data. All these arguments strengthen the reality of this possible symmetry axis.

The existence of a symmetry axis on the Stokes plane could be explained by an axisymmetric explosion where the direction of the symmetry axis is constant, but the eccentricity evolves with time. We stress that our polarimetric data can provide information about the projected geometry on the plane of the sky exclusively. Thus, we can not discard that the constant Stokes parametres of SN 2007uy could be caused by a variable eccentricity expansion when it is viewed pole-on.

\subsubsection{The robustness of the possible SN 2008D/XT 080109 symmetry axis: must we assume a constant geometry for SN 2007uy?}

One might wonder if the possible symmetry axis of SN 2008D/XT 080109 is just an artifact due to the assumption of a constant geometry for SN 2007uy. Thus we repeated the exercise explained in Sect. 3.2.2 using the original unrecalibrated
SN 2008D/XT 080109 Stokes parametres. The results are displayed in the upper panel of Fig. 1 and in Table 4. Note that the NOT Stokes parametres, could not be recalibrated, so the fit is identical to the one displayed in Table 3.

The SN 2008D/XT080109 entire data set (CAHA+VLT+ NOT $)$ is still satisfactorily fitted $\left(\chi^{2} /\right.$ d.o.f. $\left.=1.066\right)$ by a straight line. The derived orientation $\left(-49.8_{-17.4}^{+17.2} \mathrm{deg}\right)$, is fully consistent with the orientation determined based on the recalibrated Stokes parametres $\left(-42.6_{-23.3}^{+22.9} \mathrm{deg}\right)$. On the other hand, the three separated data sets yield orientations with internal consistency within $1 \sigma$, so as in Sect. 3.2.2 the inferred orientations seem to be instrumentally independent. It is also noticeable that the recalibrated Stokes parametres yield orientations $(-76.1,-64.8$, and $-54.8 \mathrm{deg}$ ) with lower internal dispersion than the unrecalibrated ones $(88.9,-64.8,-86.7 \mathrm{deg})$.

The fit quality to the VLT Stokes parametres is clearly reduced with respect to the recalibrated case, but it is still fairly satisfactory $\left(\chi^{2} /\right.$ d.o.f. $\left.=1.375\right)$. It is worthwhile to note that the major source of this $\chi^{2} /$ d.o.f. increment comes from the lack of overlap within $1 \sigma$ of the SN 2008D/XT080109 Stokes parametres corresponding to VLT3a and VLT3b (red and green circles of Fig. 1 upper panel), which appear slightly separated (by $|\Delta Q|=0.0012$ and $|\Delta U|=0.0022$, corresponding to a relative shift of $1.4 \sigma$ and $2.5 \sigma$, respectively).

One could be tempted to speculate on a possible rapid $(Q, U)$ variability in visits VLT3a and VLT3b of SN 2008D/XT 080109. However, firstly the polarimetric variation between VLT3a and VLT3b is not statistically significant, and secondly (and most importantly), this internal shift between VLT3a and VLT3b is essentially mimicked in the same visits by the behaviour of SN 2007uy. This suggests that this systematic shift present in 
both $\mathrm{SNe}$ does not reflect a real polarimetric variation, but is probably just a spurious polarization fluctuation due to slightly different GIP corrections for VLT3a and VLT3b which affects both $\mathrm{SNe}$ in a systematic way. In fact, in both visits the seven stars (identical for both visits) used for the GIP are fainter than SN 2007uy and SN 2008D/XT 080109, so they show larger photometric statistical fluctuations. Thus, when using SN 2007uy as a calibrator the Stokes shift between VLT3a and VLT3b almost disappears, overlapping within $1 \sigma$ for SN 2008D/XT 080109 (and obviously for SN 2007uy). This example illustrates the importance of using the brightest common object in the field (SN 2007uy, even brighter than SN 2008D/XT 080109) as a reference star in order to remove internal spurious shifts below $\sim 0.2 \%$ from the Stokes plane.

We conclude that, at a lower significance level, the unrecalibrated data still allow the existence of a symmetry axis for SN 2008D/XT 080109 to be plausible. Therefore, in order to suggest a symmetry axis it is not strictly necessary to assume that the SN 2007uy geometry is constant. However, this assumption strengthens its possible existence.

\subsection{Final remarks}

Maund et al. (2009) report a variation in the position angle of the He I $\lambda 5876$ line based on the comparison of their two observing epochs. Thus, we have to be cautious in explaining the origin of the possible SN 2008D/XT 080109 polarization variability. The SN 2008D/XT 080109 polarization variation suggested by the analysis of the broad-band data could not be explained, totally or partially, just by an axisymmetric expansion. We can not discard that the polarization variability could be, to some degree, caused by the changing relative strengths in the line/continuum emission, only one of which may be polarized, as discussed by Maund et al. (2009). Thus $V$-band polarimetric data alone can not resolve these potential components, and in principle only spectropolarimetry could study the impact of this, and other possible effects, on the evolution of the Stokes parametres. However, the limited time-coverage of the spectropolarimeric data (only two epochs available, covering 2 weeks around the light curve maximum) does not make it possible to derive strong conclusions about potential effects which could impact our eleven visits spanning $\sim 75$ days. We can only conclude that our data suggest a preference to an approximately axisymmetric configuration, although we can not exclude deviations from axisymmetry towards a more complex geometry, as discussed by Maund et al. (2009).

Since SN2007uy occurred earlier than SN2008D/ XT080109, we have checked to what degree a possible early fast-evolving epoch of SN 2007uy may have been missed by our observations. An inspection of the light curves of the two events shows that our data cover the rising phase, the maximum and the decay of both objects. The polarization inferred for SN2007uy in these 3 phases is consistent with being constant. Obviously, we can not discard that SN 2007uy showed polarization variability earlier than our first epoch, later than the last epoch or in the unavoidable temporal gaps between observations. So, we stress that our conclusions on SN 2007uy and SN 2008D/XT 080109 only refer to the time covered by our observations.

Under the mentioned limitations, the distribution of SN 2007uy on the Stokes plane imposes an upper limit on the variability of its polarization. First, we calculated the \pm 1 sigma confidence variability interval of $Q(U)$ around $\langle Q\rangle(\langle U\rangle)$ using the weighted variance ${ }^{10}$ of the $Q(U)$ sample as an statistical estimator. This estimator is suitable because both $Q$ and $U$ follow Gaussian distributions (as opposed to $P$ ). Then, the $(Q, U)$ weighted variances were propagated yielding a $\pm 34.145 \%$ confidence interval of $\Delta P_{ \pm 34.145 \%}={ }_{-0.09}^{+0.08 \%}$ around the SN 2007uy barycentre. Finally, using the analytic expression (A1) given in the Appendix of Wardle \& Kronberg (1974) we estimated a $\pm 45 \%$ confidence interval of $\Delta P_{ \pm 45 \%}={ }_{-0.15}^{+0.14} \%$. Thus, we estimate that over the course of our observations of SN 2007uy its total polarization signal does not change by more than $0.29 \%$ (90\% confidence interval). We double-checked that Monte Carlo methods impose similar limits on the SN 2007uy polarization variability $\left(\Delta P_{ \pm 45 \%} \sim 0.3 \%\right)$.

\section{Conclusions}

For both stellar explosions our optical polarization data seem to be dominated by the HGIP. This conclusion is supported by our $1.2 \mathrm{~mm}$ observations, performed at and around the SN 2008D/XT080109 position on NGC 2770. The $1.2 \mathrm{~mm}$ measurements are consistent with no intrinsic emission from SN 2008D/XT 080109 at this wavelength, and can be explained by the host galaxy dust emission. The $A_{V}$ inferred from our $1.2 \mathrm{~mm}$ observations is $\sim 1.2 \mathrm{mag}$ lower than the line-of-sight extinction values reported in the literature. This apparent contradiction can be solved if the NGC 2770 projected dust distribution around SN 2008D/XT 080109 is composed of dense clumps $\left(A_{V} \sim 1.2-2.5 \mathrm{mag}\right)$ with a typical size $<0.16 \mathrm{kpc}$ and a low filling factor (10-30\%) in our $1.2 \mathrm{~mm}$ beam.

As a bonus, we also report the detection of $0.65 \mathrm{mJy} / \mathrm{beam}$ at $3.3 \mathrm{~mm}$ coincident with SN 2008D/XT 08010914 days after the $\mathrm{X}$-ray outburst. This emission agrees fairly well with the light curve modeled at $95 \mathrm{GHz}$ (Soderberg et al. 2008). Furthermore an observation at $2.9 \mathrm{~mm}$ carried out on November 9.3 UT 2008 (304.8 days after the XT), imposed a $3 \sigma$ flux upper limit of $0.30 \mathrm{mJy} / \mathrm{beam}$, so we conclude that the $3.3 \mathrm{~mm}$ detection is mostly intrinsic emission from SN 2008D/XT 080109.

SN 2008D/XT 080109 seemed to be surrounded by an external thick He mantle (Mazzali et al. 2008; Modjaz et al. 2009; Soderberg et al. 2008; Tanaka et al. 2009b), in contrast to the He-poor XRF 060218/SN 2006aj (Pian et al. 2006; Soderberg et al. 2006; Sollerman et al. 2006). Given that the asphericity of core-collapse supernovae is more pronounced in the inner layers Wang \& Wheeler (2008), this could explain to some degree the modest SN 2008D/XT 080109 intrinsic polarization in comparison to XRF 060218/SN 2006aj (Gorosabel et al. 2006; Maund et al. 2007).

Notwithstanding the important HGIP, a statistical analysis of the distribution of the SN 2008D/XT 080109 Stokes parametres suggests that SN 2008D/XT 080109 could exhibit an intrinsic variable polarization component embedded in the dominant HGIP. In contrast, the SN 2007uy polarization data can be described by a constant projected geometry. This conclusion is achieved independent of the data being considered jointly or separately according to the telescope used.

\footnotetext{
10 As defined by the N.I.S.T. (National Institute of Standards and Technology; Gaithersburg, Maryland, USA, http://www .nist.gov/ index.html), $S_{Q}=\sqrt{\frac{N}{N-1} \sum_{i=0}^{N} w_{Q_{i}}\left(Q_{i}-\langle Q\rangle\right)^{2}}$, being $w_{Q_{i}}=\frac{1 / \sigma_{Q_{i}}^{2}}{\sum_{j=0}^{N} \sigma_{Q_{j}}^{2}}$, $\sigma_{Q_{i}}$ the standard deviation of $Q_{i}$, and $N$ the number of SN 2007uy visits. The expression of $S_{U}$ is analogous, replacing $Q_{i}$ with $U_{i},\langle Q\rangle$ with $\langle U\rangle$, and $\sigma_{Q_{i}}$ with $\sigma_{U_{i}}$.
} 
Assuming that the SN 2007uy projected geometry is constant in time, then the evolution of the SN 2008D/XT080109 Stokes parametres could show a symmetry axis. The same conclusion is achieved if no constant polarization is assumed for SN 2007uy, but at a lower significance level. This possible symmetry axis is also independent of the instrumentation used to perform the observations, supporting its reality. This potential axis could be approximately explained by an axisymmetric aspherical expansion with variable eccentricity. However, our broad-band polarimetric data can not exclude more complex geometric configurations, like possible deviations from axisymmetry. Our results are consistent with the SN 2008D/XT 080109 asphericity inferred from spectroscopic data (Modjaz et al. 2009; Tanaka et al. 2009a). We suggest that at least the projected, if not the intrinsic, geometry of the two explosive events could be different.

Acknowledgements. The research of J.G., A.J.C.T., M.J. and I.A. is supported by the Spanish programmes ESP2005-07714-C03-03, AYA2007-63677, AYA2007-67627-C03-03, AYA2008-03467/ESP and AYA2009-14000-C03-01. This work has been partially funded by the Spanish-German Acción Integrada HA2007-0079. AdUP acknowledges support from an ESO fellowship. A.R. and S.K. acknowledge support by DFG K1 766/11-3, P.F. and D.A.K. by the Thüringer Landessternwarte. I.A. acknowledges support by an I3P contract with the Consejo Superior de Investigaciones Científicas (CSIC). We thank M.A. Pérez Torres, C. Barceló, and L.M. Sarro for fruitful comments. The Dark Cosmology Centre is funded by the DNRF. This paper is based on data from the IRAM $30 \mathrm{~m}$ telescope, the PdB interferometer, the NOT, the VLT and the $2.2 \mathrm{~m}$ Telescope of Calar Alto. ALFOSC is owned by Instituto de Astrofísica de Andalucía (IAA) and operated at the NOT under agreement between the IAA and NBIfAFG. We thank the IRAM Director and C. Thum for providing discretionary observing time at the IRAM $30 \mathrm{~m}$ telescope. The German-Spanish Astronomical Center, Calar Alto, is jointly operated by the MPIA Heidelberg and IAA (CSIC). IRAM is supported by INSU/CNRS (France), MPG (Germany) and IGN (Spain). The VLT data were acquired by means of the ESO target of opportunity programme 080. D-0008(A). S.K. and D.A.K. acknowledge support by DFG grant K1 766/16-1. We are grateful to Dr. T. Szeifert for his suggestions on the polarimetric data reduction. We thank the anonymous referee for constructive comments.

\section{References}

Anupama, G. C., Sahu, D. K., Deng, J., et al. 2005, ApJ, 631, L125 Babu, G. J., \& Feigelson, E. D. 1996, Astrostatistics (London: Chapman \& Hall) Berger, E., \& Soderberg, A. M. 2008, GCN Circ., 7159

Bietenholz, M. F., Soderberg, A. M., \& Bartel, N. 2009, ApJ, 694, L6

Blondin, S., \& Calkins, M. 2008, Cent. Bur. Electron. Telegr., 1191

Blondin, S., Matheson, T., \& Modjaz, M. 2008, GCN Circ., 7173

Boggs, P. T., Donaldson, J. R., Byrd, R. H., \& Schnabel, R. B. 1990, ODRPACK: Software of weighted orthogonal distance regression, ACM Trans. Math. Software, 16, 348
Cardelli, J. A., Clayton, G. C., \& Mathis, J. S. 1989, ApJ, 345, 245 Chevalier, R. A., \& Fransson, C. 2008, ApJ, 683, L135

di Serego Alighieri, S. 1997, in Instrumentation for Large Telescopes, VII Canary Islands Winter School of Astrophysics, ed. J. M. Rodríguez Espinosa, A. Herrero, \& F. Sánchez (Cambridge Univ. Press), 287 Deng, J., \& Zhu, Y. 2008, GCN Circ., 7160

Domingue, D. L., Keel, W. C., Ryder, S. D., \& White, R. E. III 1999, AJ, 118, 1542

Fossati, J., Bagnulo, S., Mason, E., \& Landi Degl'Innocenti, E. 2007, in The Future of Photometric, Spectrophotometric and Polarimetric Standardization, ed. C. Sterken, ASP Conf. Ser., 364, 503

González-Delgado, R., \& Pérez, E. 1997, ApJS, 108, 199

Gorosabel, J., Larionov, V., Castro-Tirado, A. J., et al. 2006, A\&A, 459, L33

Höflich, P. 1991, A\&A, 246, 481

Kasen, D., Nugent, P., Wang, L., et al. 2003, ApJ, 593, 788

Kong, A. K. H., \& Maccarone, T. J. 2008, Cent. Bur. Electron. Telegr., 1355

Kramer, C., Alves, J., Lada, C., et al. 1998, A\&A, 329, L33

Lemmerling, P., \& van Huffel, S. 2002, Total least squares and errors-in-variables modeling: analysis, algorithms and applications (Dordretch, Boston: Kluwer Academic)

Li, L.-X. 2008, MNRAS, 388, 603

Malesani, D., Fynbo, J. P. U., Hjorth, J., et al. 2009, ApJ, 692, L84

Maund, J. R., Wheeler, J. C., Patat, F., et al. 2007, A\&A, 475, L1

Maund, J. R., Wheeler, J. C., Baade, D., et al. 2009, ApJ, 705, 1139

Mazzali, P., Valenti, S., Della Valle, M., et al. 2008, Science, 321, 1185

Modjaz, M., \& Falco, E. 2005, IAU Circ., 8461

Modjaz, M., Krishner, R., Challis, P., \& Berlind, P. 2005, IAU Circ., 8522

Modjaz, M., Li, W., Butler, N., et al. 2009, ApJ, 702, 226

Nakano, S., Kadota, K., Itagati, K., \& Corelli, P. 2008, IAU Circ., 8908

Pian, E., Mazzali, P. A., Masetti, N., et al. 2006, Nature, 442, 1011

Press, W. H., Teukolsky, S. A., Vetterling, W. T., \& Flannery, B. P. 1992, Numerical Recipes, 2nd ed. (Cambridge Univ. Press)

Pugh, H., Li, W., Manzini, F., \& Behrend, R. 2004, IAU Circ., 8452

Rosolowsky, E., Keto, E., Matsushita, S., \& Willner, S. P. 2007, ApJ, 661, 830

Serkowski, K., Mathewson, D. S., \& Ford, V. L. 1975, ApJ, 196, 261

Schlegel, D. J., Finkbeiner, D. P., \& Davis, M. 1998, ApJ, 500, 525

Schmidt, G. D., Elston, R., \& Lupie, O. L. 1992, AJ, 104, 1563

Soderberg, A. M., Kulkarni, S. R., Nakar, E., et al. 2006, Nature, 442, 1014

Soderberg, A. M., Berger, E., Page, K. L., et al. 2008, Nature, 453, 469

Sollerman, J., Jaunsen, A. O., Fynbo, J. P. U., et al. 2006, A\&A, 454, 503

Tanaka, M., Yamanaka, M., Maeda, K., et al. 2009a, ApJ, 700, 1680

Tanaka, M., Tominaga, N., Nomoto, K., et al. 2009b, ApJ, 692, 1131

Thöne, C., Michalowski, M. J., Leloudas, G., et al. 2009, ApJ, 698, 1307

Tominaga, N., Tanaka, M., Nomoto, K., et al. 2005, ApJ, 633, L97

Turnshek, D. A., Bohlin, R. C., Williamson, R. L. II, et al. 1990, AJ, 99, 1243

Valenti, S., Fugazza, D., Maiorano, D., et al. 2008, GCN Circ., 7171

Wang, L., \& Baade, D., 2005, IAU Circ., 8521

Wang, L., \& Wheeler, J. C. 2008, ARA\&A, 46, 433

Wang, L., Howell, D. A., Höflich, P., \& Wheeler, J. C. 2001, ApJ, 550, 1030

Wardle, J. F. C., \& Kronberg, P. P. 1974, ApJ, 194, 249

Whittet, D. C. B., Martin, P. G., Hough, J. H., et al. 1992, ApJ, 386, 562

Wolff, M. J., Nordsieck, K. H., \& Nook, M. A. 1996, AJ, 111, 856

$\mathrm{Xu}$, D., Watson, D., Fynbo, J. P. U., et al. 2008, in COSPAR Plenary Meeting, 37th COSPAR Scientific Assembly, 3512 